\title{
The Convex Hull of Rational Plane Curves
}

\author{
Gershon Elber \\ Department of Computer Science, Technion, IIT, Haifa 32000, Israel \\ Myung-Soo Kim \\ Department of Computer Engineering, Seoul National University, Seoul 151-742, Korea
}

and

Hee-Seok Heo

Department of Computer Science, POSTECH, Pohang 790-784, Korea

Received October 5, 1999; revised April 19, 2001; accepted May 22, 2001

\begin{abstract}
We present an algorithm that computes the convex hull of multiple rational curves in the plane. The problem is reformulated as one of finding the zero-sets of polynomial equations in one or two variables; using these zero-sets we characterize curve segments that belong to the boundary of the convex hull. We also present a preprocessing step that can eliminate many redundant curve segments. (c) 2001 Academic Press

Key Words: convex hull; point-curve tangent; bi-tangent; curve-curve tangent; zero-set finding; rational curve; B-spline; symbolic computation.
\end{abstract}

\section{INTRODUCTION}

Many geometric problems have solutions that are simpler for convex objects than for general nonconvex objects [13]. For example, interference among convex polygons can be detected more efficiently than among arbitrary simple polygons, and so convex hulls are commonly used in preprocessing for interference tests: when the convex hulls do not intersect, there can be no interference among the given polygons. Applications of this sort have motivated the development of several optimal algorithms for computing the convex hull of a simple polygon in linear time [6,8].

There are a few theoretical algorithms for computing the convex hull of a planar curved object $[1,3,14]$. These algorithms are optimal in the sense that their time complexity is $O(n)$, where $n$ is the number of curved edges in an input object. (Each algorithm is based on a different definition of "curved edge"; see the Refs. $[1,3,14]$ for more details.) Nevertheless, each step of these convex hull computations is based on expensive procedures that compute: 
(i) the tangent line from a point to a curved edge and (ii) the common tangent line of two curved edges. In practice, however, many planar curved objects are constructed out of a small number of freeform curves, each of which may be quite complicated; in such cases, the efficiency and robustness of computing tangent lines is dominant, and combinatorial complexity as a function of $n$ is not an appropriate measure of performance.

The boundary of the convex hull of a curve segment $C$ consists of subsegments of $C$ as well as line segments that support $C$ at two different locations. (A line supports a curve $C$ if and only if it touches $C$ at some point(s) and the curve $C$ is totally contained in the half-plane bounded by the line.) The curve $C$ may be supported by a line at: (i) both end points, (ii) one end point and one interior point, or (iii) two interior points. It is trivial to compute the line passing through both end points of $C$; a line through a fixed point and tangent to $C$ is called a point-curve tangent; and a line tangent to $C$ at two different interior points is called a bi-tangent. The supporting lines of types (ii) and (iii) are, respectively, the point-curve tangents and bi-tangents of $C$.

Point-curve tangents may be computed by solving a polynomial equation in one variable; the computation of bi-tangents can be formulated as a system of two polynomial equations in two variables, which can be solved using existing techniques $[10,11,15]$. In this paper, we employ a simple technique that represents the polynomial equations using B-spline basis functions. The convex hull property of the B-spline representation then provides a robust, yet reasonably efficient, procedure that computes all solutions based on an adaptive subdivision scheme [5].

Previous algorithms $[7,9,12]$ for computing common tangent lines required each curve to be segmented into certain simple pieces such as convex and monotone segments, which is cumbersome and time-consuming for high-degree curves. In this paper, we take a general approach that needs no special structure for the input curves and imposes no limitation on their degrees: we assume only that the input curves are planar, rational, piecewise $C^{1}$ continuous, and contain no straight line segments. Once the problem has been reformulated in terms of B-spline polynomial equations, a generic zero-set finder is used to compute all the solutions of these equations.

The zero-set approach provides a simple way of characterizing the curve segments that appear on the boundary of the convex hull. Assume that a curve point $C\left(t_{0}\right)$ is on the boundary of the convex hull. The tangent line at this point intersects the curve at no point other than $C\left(t_{0}\right)$. This condition can be formulated by complementing the projection of a zero-set.

The rest of this paper is organized as follows. In Section 2, we present preliminary algorithms for computing the point-curve tangents, bi-tangents, and common tangents for rational curves in the plane. In Section 3, we describe the main algorithm of this paper, which computes the convex hull of a rational curve and that of multiple rational curves. A technique for eliminating many redundant curve segments is introduced in Section 4. Section 5 concludes this paper.

\section{PRELIMINARIES}

To begin, we will consider how to compute: (i) the tangent lines from a point $P$ to a piecewise rational curve $C$, (ii) all lines tangent to $C$ at two different locations, and (iii) all lines tangent to two different curves $C_{1}$ and $C_{2}$ simultaneously. We reduce each procedure into a zero-set finding problem in one or two variables. 


\subsection{Computing Point-Curve Tangents}

Let $P=\left(p_{x}, p_{y}\right)$ be a fixed point and

$$
C(t)=\left(\frac{x(t)}{w(t)}, \frac{y(t)}{w(t)}\right)
$$

be a regular $C^{1}$-continuous rational curve of degree $d$ in the $x y$-plane. The line $L_{P, C\left(t_{0}\right)}$ passing through $P$ and $C\left(t_{0}\right)$ is tangent to the curve $C(t)$ at the point $C\left(t_{0}\right)$ if and only if the vector $C\left(t_{0}\right)-P$ is orthogonal to the normal vector $N\left(t_{0}\right)=w^{\prime}\left(t_{0}\right)\left(y\left(t_{0}\right),-x\left(t_{0}\right)\right)$ $-w\left(t_{0}\right)\left(y^{\prime}\left(t_{0}\right),-x^{\prime}\left(t_{0}\right)\right)$. This condition can be formulated as a polynomial equation of degree $2 d-2$ in one variable $t$

$$
\begin{aligned}
\mathcal{F}_{1}(t)= & \langle C(t)-P, N(t)\rangle \\
= & \left(x^{\prime}(t) y(t)-x(t) y^{\prime}(t)\right) \\
& +p_{x}\left(w(t) y^{\prime}(t)-w^{\prime}(t) y(t)\right)+p_{y}\left(x(t) w^{\prime}(t)-x^{\prime}(t) w(t)\right) \\
= & 0,
\end{aligned}
$$

which has at most $2 d-2$ real roots. In Fig. 1a, the curve $C(t)$ is drawn in solid black and all tangent lines to $C(t)$ from a fixed point $P$ are drawn in gray. The function $\mathcal{F}_{1}(t)$ of Eq. (1) is shown in Fig. 1b. Both $C(t)$ and $\mathcal{F}_{1}(t)$ are represented as B-spline curves.

\subsection{Computing Bi-tangents and Curve-Curve Tangents}

When the point $P=\left(p_{x}, p_{y}\right)$ is located on the same curve $C(r)$, Eq. (1) can be reformulated as

$$
\begin{aligned}
\mathcal{F}_{2}(r, t)= & w(r)\langle C(t)-C(r), N(t)\rangle \\
= & w(r)\left(x^{\prime}(t) y(t)-x(t) y^{\prime}(t)\right) \\
& +x(r)\left(w(t) y^{\prime}(t)-w^{\prime}(t) y(t)\right)+y(r)\left(x(t) w^{\prime}(t)-x^{\prime}(t) w(t)\right) \\
= & 0 .
\end{aligned}
$$
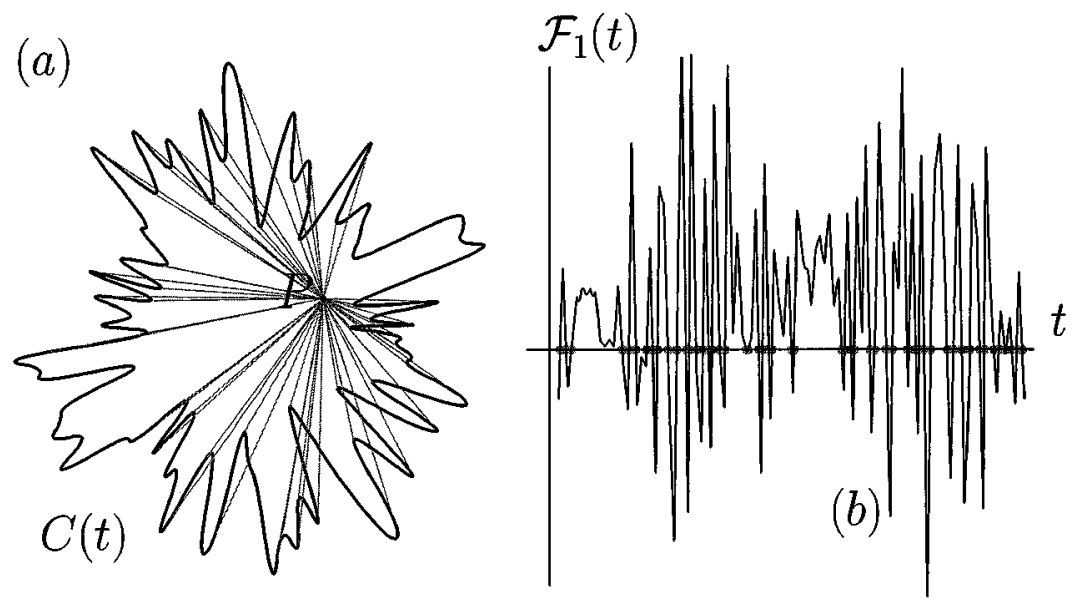

FIG. 1. $C(t)$ is a quadratic periodic B-spline curve with 103 control points: (a) shows all tangents lines from a point $P$ to a curve $C(t)$; and in (b) the function $\mathcal{F}_{1}(t)$ is shown together with all the solutions of Eq. (1). 
Additionally, when the line $L_{C(t), C(r)}$ is parallel to both $C^{\prime}(t)$ and $C^{\prime}(r)$, we have the following two simultaneous equations in two variables

$$
\begin{aligned}
\mathcal{F}_{2}^{1}(r, t) & =w(r)\langle C(t)-C(r), N(t)\rangle=0, \\
\mathcal{F}_{2}^{2}(r, t) & =w(t)\langle C(r)-C(t), N(r)\rangle=0 .
\end{aligned}
$$

Let $\overline{\mathcal{F}}_{2}^{1}$ and $\overline{\mathcal{F}}_{2}^{2}$ denote the zero-sets of the above two equations, respectively:

$$
\overline{\mathcal{F}}_{2}^{1}=\left\{(r, t) \mid \mathcal{F}_{2}^{1}(r, t)=0\right\} \quad \text { and } \quad \overline{\mathcal{F}}_{2}^{2}=\left\{(r, t) \mid \mathcal{F}_{2}^{2}(r, t)=0\right\} .
$$

Note that Eqs. (3) and (4) are symmetric with respect to the variables $r$ and $t: \mathcal{F}_{2}^{1}(t, r)=$ $\mathcal{F}_{2}^{2}(r, t)$. Thus, the zero-sets $\overline{\mathcal{F}}_{2}^{1}$ and $\overline{\mathcal{F}}_{2}^{2}$ are symmetric about the diagonal line $r=t$. When one zero-set is constructed, the other can be simply obtained as its reflection about the diagonal line. The simultaneous solutions of Eqs. (3) and (4) are given as the elements in the intersection of two zero-sets $\overline{\mathcal{F}}_{2}^{1} \cap \overline{\mathcal{F}}_{2}^{2}$.

Figure 2 shows an example. In Fig. 2a the function $\mathcal{F}_{2}^{1}(r, t)$ is shown as a scalar B-spline surface. The zero-set $\overline{\mathcal{F}}_{2}^{1}$ is computed; its reflection about the diagonal line $r=t$ yields

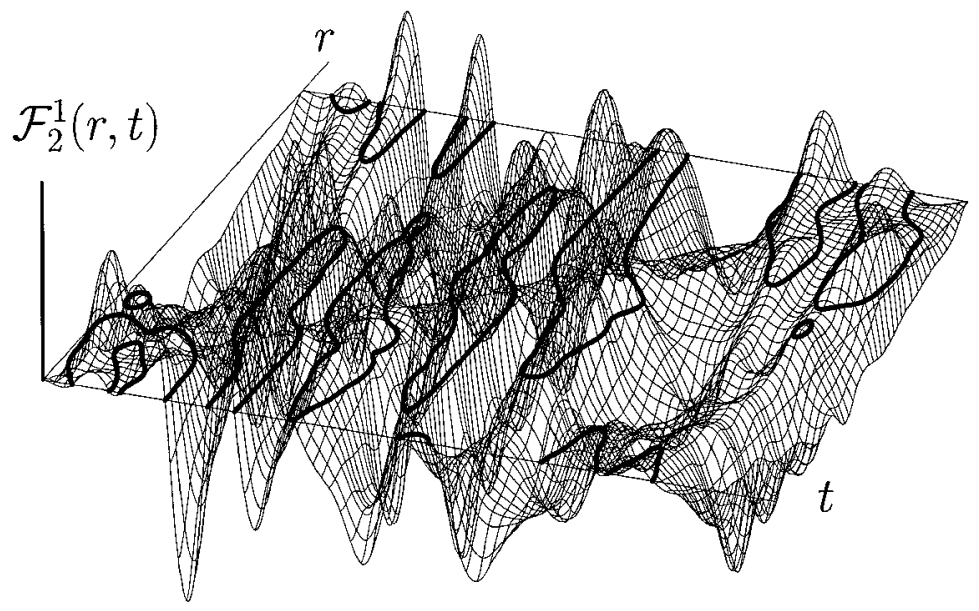

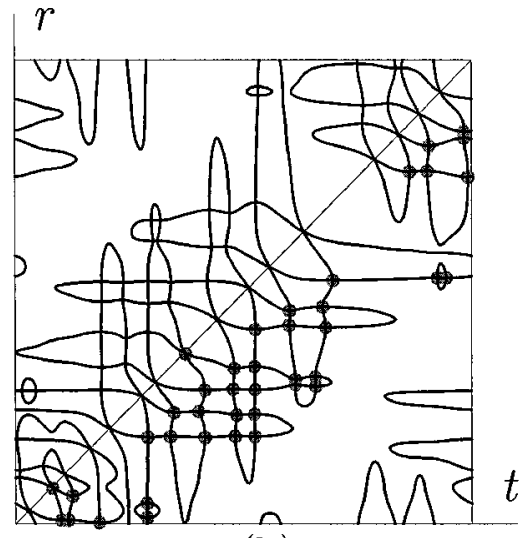

(b)

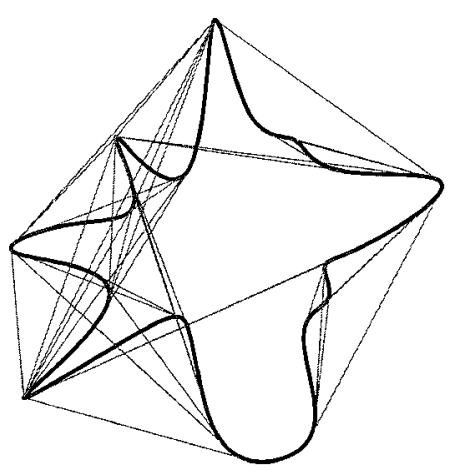

(c)

FIG. 2. The two zero-sets of Eqs. (3) and (4) are intersected to yield the pairs $(r, t)$ that admit common tangent lines to $C$ : in (a), the function $\mathcal{F}_{2}^{1}(r, t)$ is shown along with its zero-set, $\overline{\mathcal{F}}_{2}^{1}$; in (b), the intersection points of $\overline{\mathcal{F}}_{2}^{1} \cup \overline{\mathcal{F}}_{2}^{2}$ (below the diagonal line $r=t$ ) are drawn in gray; finally, in (c), all the computed bi-tangents are shown. 
the other zero-set $\overline{\mathcal{F}}_{2}^{2}$. Their intersection $\overline{\mathcal{F}}_{2}^{1} \cap \overline{\mathcal{F}}_{2}^{2}$ provides all $(r, t)$-pairs of parametric locations that admit bi-tangent lines. Due to the symmetry, only the solutions below the diagonal line $r=t$ need to be considered. Figure 2c shows the original curve itself with all its bi-tangent lines. All pairs $(r, t)$ on the diagonal line $r=t$ clearly satisfy Eqs. (3) and (4). In Fig. 2b, it is easy to see that the whole diagonal $r=t$ is contained in the zeroset. Nevertheless, we do not consider solutions above the diagonal since they are of no practical use.

If $C_{1}(t)$ and $C_{2}(r)$ are two regular $C^{1}$-continuous rational curves in the $x y$-plane, their common tangent lines can be found by replacing $C(t)$ by $C_{1}(t)$ and $C(r)$ by $C_{2}(r)$ in the above equations.

\section{THE CONVEX HULL OF FREEFORM CURVES}

Assuming a "black box" that computes all bi-tangents, the boundary of the convex hull of a curve $C(t)$ (denoted as $\mathcal{C H}(C)$ ) can be constructed by selecting the appropriate curve segments of $C(t)$ and some bi-tangent line segments. When $C(t)$ is an open curve, the boundary of the convex hull may also contain at most four point-curve tangent lines (from the end points of $C(t)$ ). For computational efficiency, it is important to construct only the bi-tangents and point-curve tangents that appear on the boundary of the convex hull. Without loss of generality, we may assume that the curve $C(t)$ is $C^{1}$-continuous by splitting it into many pieces if necessary. It is quite straightforward to merge two convex hulls (see Fig. 3). Thus the convex hull of multiple $C^{1}$-continuous curves can be constructed by first computing the convex hull of each $C^{1}$-continuous curve and then merging them into the convex hull of all curves. (A more efficient method will be discussed later.)

In general, the total number of bi-tangents for a rational curve $C(t)$ of degree $d$ is $O\left(d^{2}\right)$. On the other hand, there are only $O(d)$ inflection points, which means that the curve $C(t)$ has $O(d)$ convex segments. Thus, there are at most $O(d)$ convex segments on the boundary of the convex hull. Note that each convex curve segment in $\mathcal{C H}(C)$ is adjacent to at most two bi-tangents. Consequently, there are at most $O(d)$ bi-tangents that can appear in $\mathcal{C H}(C)$. Moreover, there are at most four point-curve tangents.

Consider the two points $C\left(t_{1}\right)$ and $C\left(t_{2}\right)$ in Fig. 4. The point $C\left(t_{1}\right)$ is in $\mathcal{C H}(C)$ since the curve $C(t)$ is completely on one side of the tangent line at $C\left(t_{1}\right)$. On the other hand, $C\left(t_{2}\right)$ is not in $\mathcal{C H}(C)$. The tangent line at $C\left(t_{2}\right)$ intersects the curve transversally at $C\left(t_{3}\right)$ and $C\left(t_{4}\right)$; thus the curve $C(t)$ cannot be completely on one side of the tangent line. When a tangent line has no transversal intersection, the curve $C(t)$ is completely on one side of the tangent

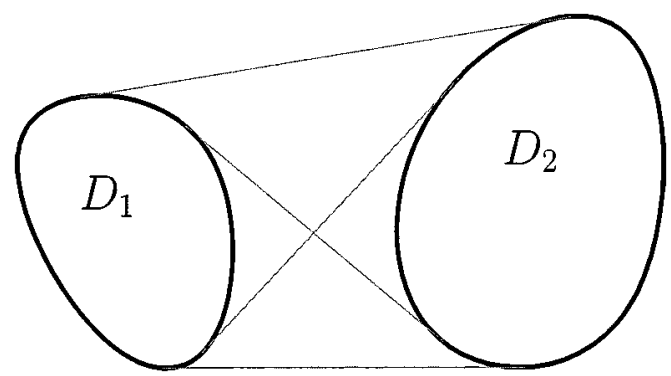

FIG. 3. Two convex closed curves can have at most four bi-tangent lines. 


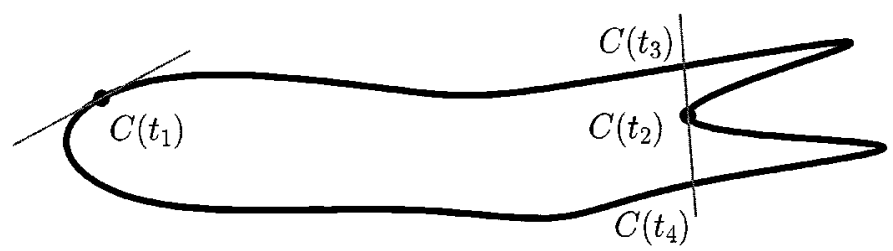

FIG. 4. Point $C\left(t_{1}\right)$ is on $\mathcal{C H}(C)$ while point $C\left(t_{2}\right)$ is not. Note that $C(t)$ is completely on one side of the tangent line at $C\left(t_{1}\right)$; in contrast, there are some segments of the curve on both sides of the tangent at $C\left(t_{2}\right)$.

line and the tangent point is on the boundary of the convex hull. This simple observation can be summarized as follows:

LEMMA 1. A curve interior point $C\left(t_{1}\right) \in \mathcal{C H}(C)$ if and only if the tangent line of $C(t)$ at $C\left(t_{1}\right)$ has no transversal intersection with $C(t)$.

Remark. We have not considered the end points of an open curve $C(t)$ in the above lemma. Each end point may be considered as a semicircle with radius zero. Any line passing through the end point may be considered as a tangent line to the semicircle. Thus an end point of the curve $C(t)$ is in $\mathcal{C H}(C)$ if and only if there is at least one line that passes through the end point but does not intersect the curve transversally at an interior point. In Fig. 5, the two end points of $C(t)$ share the same tangent line (i.e., the vertical line passing through them); however, $P_{1} \in \mathcal{C H}(C)$, whereas $P_{2} \notin \mathcal{C H}(C)$. The horizontal line passing through $P_{1}$ intersects $C(t)$ at no other point; however, any line passing through $P_{2}$ intersects $C(t)$ transversally at some other point(s).

The condition $\mathcal{F}_{2}(r, t)=0$ of Eq. (2) means that the tangent line at $C(t)$ intersects the curve at $C(r)$ (transversally or tangentially). The set of curve points, at each of which the tangent line intersects the curve at no other points, is defined as

$$
\mathcal{C H}^{o}(C)=\left\{C(t) \mid \mathcal{F}_{2}(r, t) \neq 0, \forall r \neq t\right\}
$$

This set $\mathcal{C} \mathcal{H}^{o}(C)$ is clearly a subset of the boundary of the convex hull and it is also a subset of the curve $C(t)$ itself:

$$
\mathcal{C H}^{o}(C) \subset \mathcal{C H}(C) \cap C .
$$

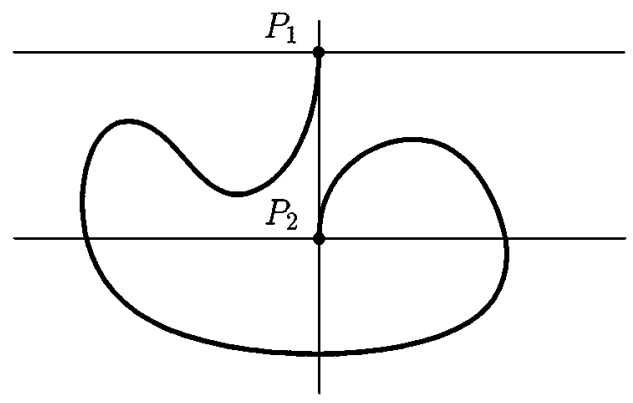

FIG. 5. An open curve. 
The difference $\mathcal{C H}(C) \cap C \backslash \mathcal{C H}^{o}(C)$ contains some extra points such as: (i) the end points of each connected curve segment of $\mathcal{C H}^{o}(C)$ and, sometimes, (ii) the end points of $C(t)$. (We assume that each supporting line of $C(t)$ is tangent to $C(t)$ at no more than two curve points; thus all isolated points of $\mathcal{C H}(C) \cap C$ must be end points of $C(t)$.)

We sort the curve segments in $\mathcal{C H}^{o}(C)$ according to their normal (or tangent) angles. If there is a gap between the normal angles of two consecutive curve segments in this sorted list, then an end point of $C(t)$ must correspond to the gap since it is a global extreme point in the missing normal directions (see point $P_{1}$ in Fig. 5). In this case, we need to insert the end points of $C(t)$ into the sorted list of curve segments of $\mathcal{C H}^{o}(C)$. (Note that we may consider each curve end point as a degenerate semicircle of radius zero.) By connecting each pair of two consecutive segments (in this sorted order) by their common tangent line segment, we can construct $\mathcal{C H}(C)$. Thus, the above set $\mathcal{C H} \mathcal{H}^{\circ}(C)$ of convex curve segments and the end points of $C(t)$ define $\mathcal{C H}(C)$ completely and uniquely.

The diagonal line $r=t$ is contained in the zero-set of $\mathcal{F}_{2}(r, t)$. Moreover, one can show that $(r-t)^{2}$ is a factor of $\mathcal{F}_{2}(r, t)$. If $\hat{\mathcal{F}}_{2}(r, t)=(r-t)^{-2} \mathcal{F}_{2}(r, t)$, then $\hat{\mathcal{F}}_{2}(r, t)$ has no more factors of $(r-t)$ (we omit the proof here). Thus the zero-set of $\hat{\mathcal{F}}_{2}(r, t)$ characterizes the set $\mathcal{C} \mathcal{H}^{o}(C)$ of Eq. (6). Consider the projection of the zero-set of $\hat{\mathcal{F}}_{2}(r, t)$ on to the $t$-axis. The intervals on the $t$-axis that are "uncovered" (in the projection) correspond to the curve segments of $\mathcal{C H}^{o}(C)$. Figure $6 \mathrm{c}$ shows the convex hull of a closed curve; the function $F_{2}(r, t)$ is shown in Fig. 6a, and its zero-set (except the diagonal line $r=t$ ) is shown in Fig. 6b along with its projection on to the $t$-axis.

Figure 7a shows all tangent lines from a point $P$ to an open curve $C(t)$. All bi-tangent lines of $C(t)$ are presented in Fig. 7b. Finally, all segments of $C(t)$ that appear in $\mathcal{C H}(C)$ are displayed in Fig. 7c. Figure 8 shows the same technique applied to a self-intersecting curve.

\section{TRIMMING REDUNDANT CURVE SEGMENTS}

Given a set of curve segments $\left\{C_{i}\right\}$ in the plane, each boundary point on the convex hull must be a global extreme point (among all curve points) in the normal direction of the convex hull at the boundary point. Assume that we have selected $2^{k}$ normal directions: $N_{j}^{k}=\left(\cos \frac{2 \pi j}{2^{k}}, \sin \frac{2 \pi j}{2^{k}}\right)$, for $j=0, \ldots, 2^{k}-1$. Let $p_{j}$ be the extreme point (among all curve points of $\left.\left\{C_{i}\right\}\right)$ in the direction of $N_{j}^{k}$; that is, we have $\left\langle p_{j}, N_{j}^{k}\right\rangle=\max \left\{\left\langle p, N_{j}^{k}\right\rangle \mid\right.$ $p \in \cup C_{i}$. . (See Figs. 9a-9d for examples of such normal directions and the corresponding extreme points in these directions; note that $N_{j}^{k}=N_{2 j}^{k+1}$ and thus the set of normal directions $\left\{N_{j}^{k}\right\}$ is properly contained in the set $\left\{N_{j}^{k+1}\right\}$.) If there is more than one such point, we consider $p_{j}$ to be the line segment connecting all of them. The closed convex polygon $P_{k}\left(=p_{0}, p_{1}, \ldots, p_{2^{k}-1}, p_{0}\right)$ thus defines a sequence of gradually expanding convex polygons $\left\{P_{k}\right\}$ that converges to $\mathcal{C H}\left(\cup C_{i}\right)$, the boundary of the convex hull of the curve segments $\left\{C_{i}\right\}$.

Let $\mathcal{I}\left(P_{k}\right)$ denote the interior region of the convex polygon $P_{k}$. Any point of $C_{i}$ contained in the open region $\mathcal{I}\left(P_{k}\right)$ cannot be on the boundary of the convex hull $\mathcal{C H}\left(\cup C_{i}\right)$. Let $\left\{C_{i}^{k}\right\}$ denote the curve segments that are left after trimming based on the convex region $\mathcal{I}\left(P_{k}\right)$. For a sufficiently large $k$, the set $\left\{C_{i}^{k}\right\}$ mostly consists of curve segments that actually appear on the boundary of the convex hull. However, it is difficult to determine such a value of $k$ a priori. Thus we may start with a small value of $k$ (say, $k=3$ or 4 ), compute $2^{k}$ extreme 


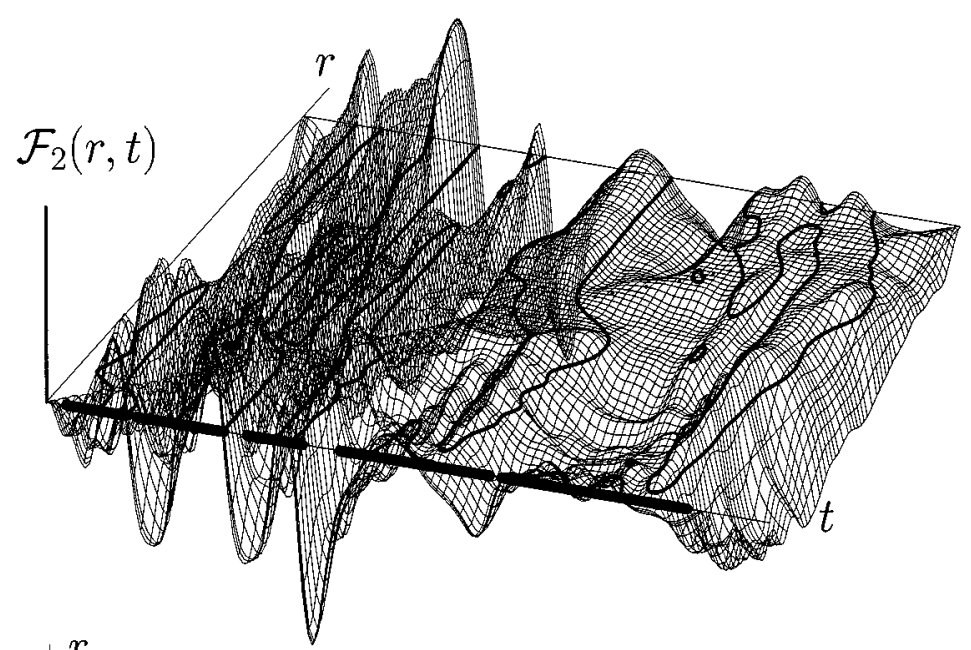

(a)

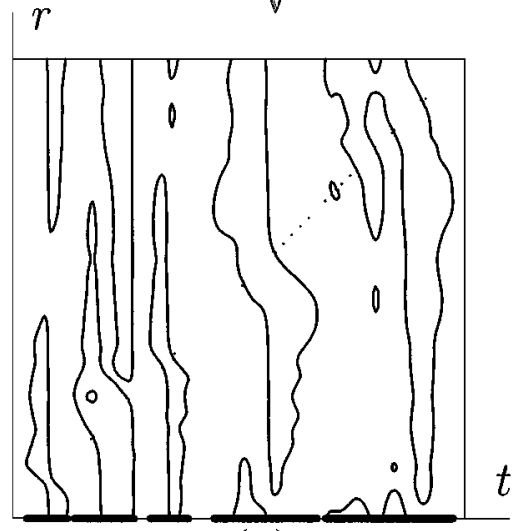

(b)

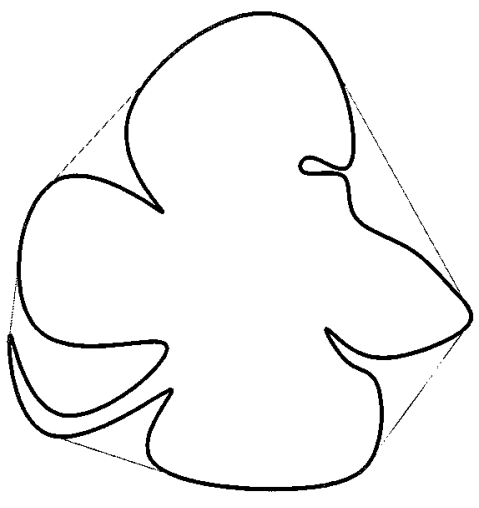

(c)

FIG. 6. The convex hull of a cubic periodic B-spline curve with 33 control points: (a) the function $\mathcal{F}_{2}(r, t)$ is shown along with its zero-set (except the diagonal line $r=t$ ); (b) the zero-set contours (except the diagonal line $r=t$ ) are shown in the $(t, r)$-plane (the contours projected on to the $t$-axis provide the domain of the curve, which is not part of its convex hull); and (c) shows the final convex hull.

points, and trim out the input curve segments $\left\{C_{i}\right\}$ using the convex region $\mathcal{I}\left(P_{k}\right)$. After that, we compute $\mathcal{C H}\left(C_{i}^{k}\right)$, the boundary of the convex hull of each trimmed curve segment $C_{i}^{k}$, and merge them into $\mathcal{C H}\left(\cup C_{i}\right)$, the boundary of the convex hull for all curve segments $\left\{C_{i}\right\}$. Figure 9 shows a closed Jordan curve and its expanding convex polygons $P_{k}$, for

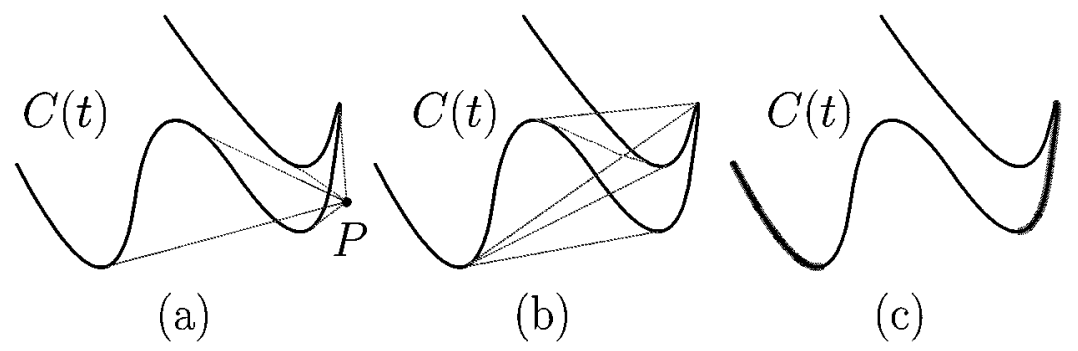

FIG. 7. $C(t)$ is a quadratic B-spline curve with open end conditions and eight control points: (a) shows all tangent lines from a point $P$ to a curve $C(t)$; (b) shows all bi-tangent lines of $C(t)$; and (c) shows all segments of $C$ that appear in $\mathcal{C H}(C)$. 


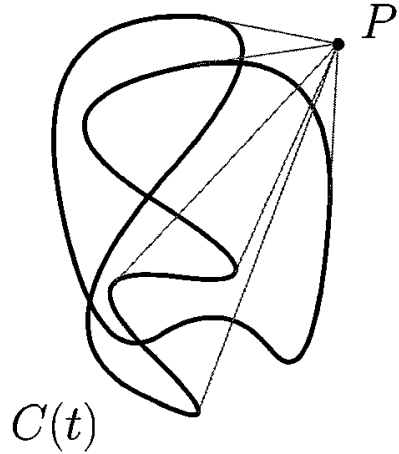

(a)

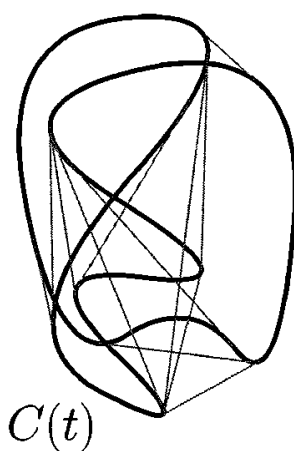

(b)

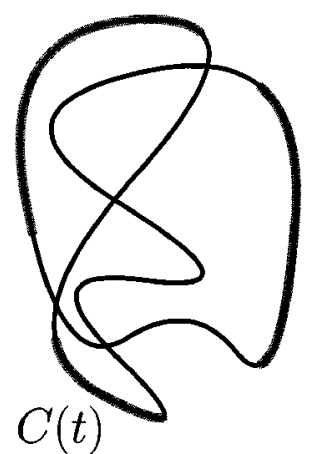

(c)

FIG. 8. $C(t)$ is a quadratic B-spline curve with 13 control points: (a) shows all tangent lines from a point $P$ to a curve $C(t)$; (b) shows all bi-tangent lines of $C(t)$; and (c) shows all segments of $C$ that appear in $\mathcal{C H}(C)$.

$k=1,2,3,4,5$. In Figs. 9a-9e, the trimmed curves $\left\{C_{i}^{k}\right\}$ are drawn as bold lines. The convex hull itself is shown in Fig. 9f.

Figure 10 shows a curve with a complicated shape. In Figs. 10a-10e, the convex polygons $P_{k}(k=1,2,3,4,5)$ are shown in thin lines and the trimmed subsegments of $C(t)$ in the

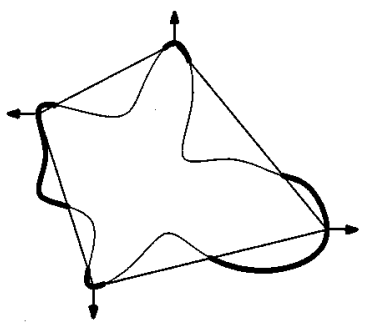

(a) $k=1$

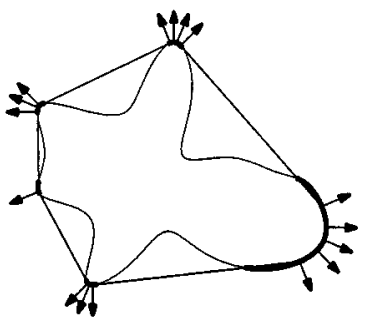

(c) $k=3$

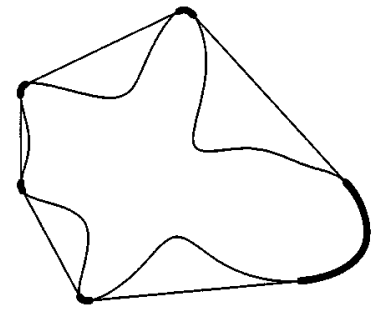

(e) $k=5$

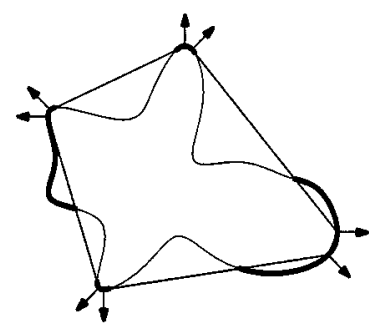

(b) $k=2$

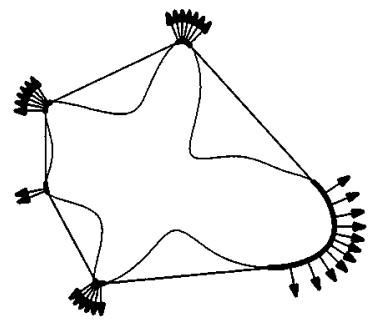

(d) $k=4$

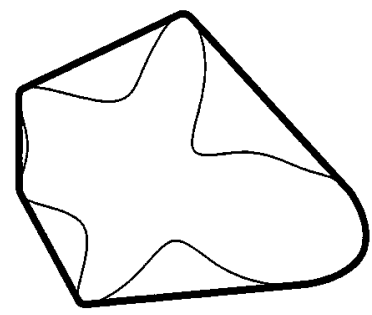

(f) convex hull

FIG. 9. Expanding convex polygons and the convex hull. 


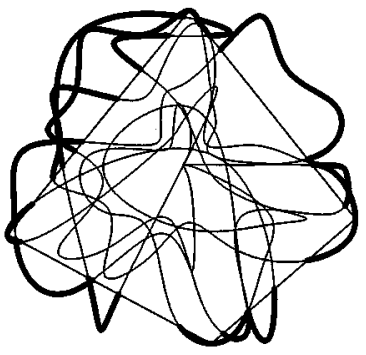

(a) $k=1$

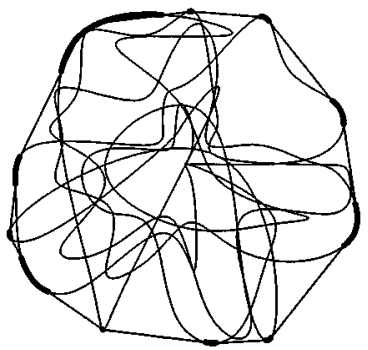

(c) $k=3$

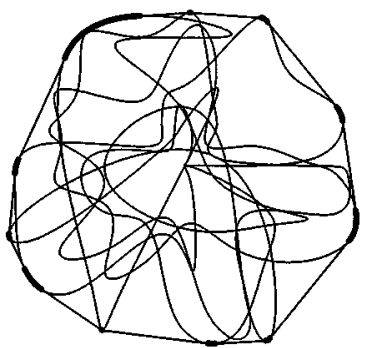

(e) $k=5$

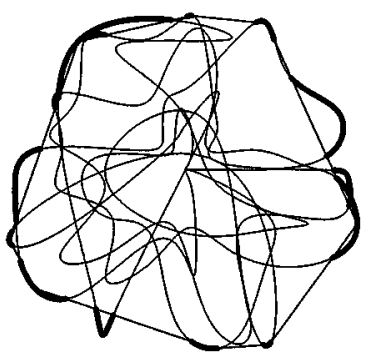

(b) $k=2$

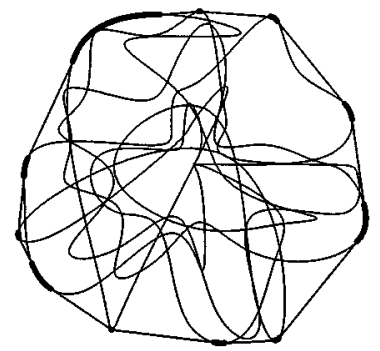

(d) $k=4$

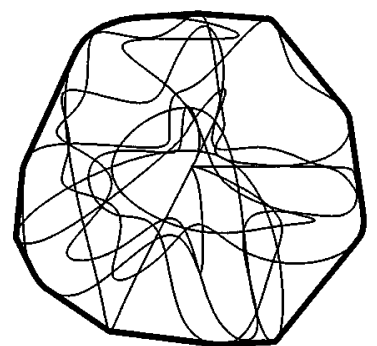

(f) convex hull

FIG. 10. Convex hull of a complicated curve.

exterior of $P_{k}$ are shown in bold lines. Consider a half-plane that contains a convex polygon $P_{k}=\left(p_{0}, p_{1}, \ldots, p_{2^{k}-1}, p_{0}\right)$ and is bounded by an infinite line $\overline{p_{i} p_{i+1}}$. For a sufficiently large $k$ (e.g., for $k \geq 3$, as in Fig. 10), there are at most two trimmed subsegments of $C(t)$ that may lie outside this half-plane. Let $C_{i}(t)$ and $C_{i+1}(r)$ denote two such trimmed subsegments of $C(t)$.

The set $\mathcal{C H}(C) \cap C_{i}$ is characterized by

$$
\mathcal{C H}^{o}\left(C_{i}\right)=\left\{C_{i}(t) \mid \mathcal{F}_{2}^{i}(s, t) \neq 0 \wedge \mathcal{F}_{3}^{i}(r, t)>0, \forall s, r \neq t\right\}
$$

In the above equation, $\mathcal{F}_{2}^{i}(s, t)$ and $\mathcal{F}_{3}^{i}(r, t)$ are formulated by modifying Eq. (2) as

$$
\begin{aligned}
& \mathcal{F}_{2}^{i}(s, t)=w_{i}(s)\left\langle C_{i}(t)-C_{i}(s), N_{i}(t)\right\rangle, \\
& \mathcal{F}_{3}^{i}(r, t)=w_{i+1}(r)\left\langle C_{i}(t)-C_{i+1}(r), N_{i}(t)\right\rangle,
\end{aligned}
$$

where we assume that $w_{i}(s)>0$ and $w_{i+1}(r)>0$. The condition $\mathcal{F}_{2}^{i}(s, t) \neq 0$ means that line tangent to $C_{i}(t)$ intersects the curve $C_{i}(t)$ at no other point $C_{i}(s), s \neq t$; moreover, 


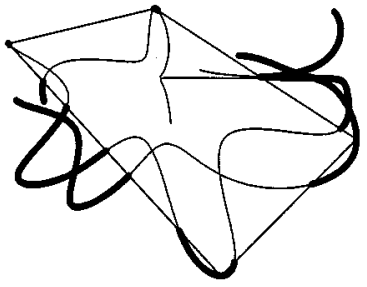

(a) $k=1$

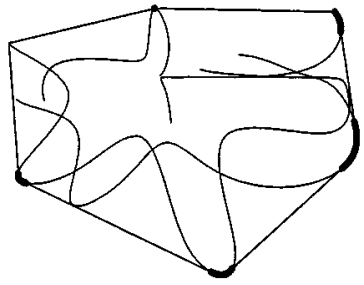

(c) $k=3$

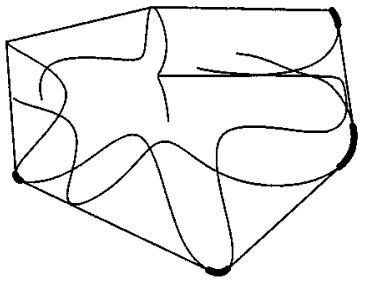

(e) $k=5$

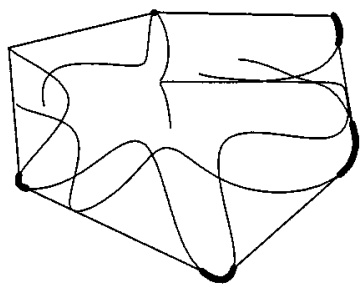

(b) $k=2$

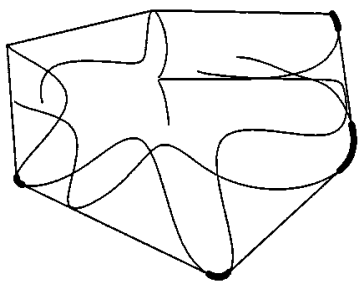

(d) $k=4$

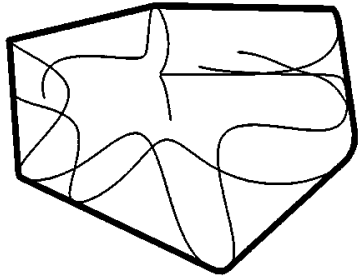

(f) convex hull

FIG. 11. Convex hull of multiple curves.

$\mathcal{F}_{3}^{i}(r, t)>0$ means that the curve segment $C_{i+1}(r)$ is totally contained in the interior of the half-plane that contains the convex polygon $P_{k}$ and is bounded by the line tangent to $C_{i}(t)$. By switching the roles of $C_{i}(t)$ and $C_{i+1}(r)$, we can compute the subsegment of $C_{i+1}$ that contributes to $\mathcal{C H}(C)$.

When an end point $C\left(t_{0}\right)$ of $C(t)$ is located outside the half-plane bounded by $\overline{p_{i} p_{i+1}}$, the set $\mathcal{C H}(C) \cap C_{i}$ is characterized by

$$
\mathcal{C H}^{o}\left(C_{i}\right)=\left\{C_{i}(t) \mid \mathcal{F}_{1}^{i}(t)>0 \wedge \mathcal{F}_{2}^{i}(s, t) \neq 0 \wedge \mathcal{F}_{3}^{i}(r, t)>0, \forall s, r \neq t\right\},
$$

where $\mathcal{F}_{1}^{i}(t)$ is formulated by modifying Eq. (1) as

$$
\mathcal{F}_{1}^{i}(t)=\left\langle C_{i}(t)-C_{i}\left(t_{0}\right), N(t)\right\rangle,
$$

where $C_{i}\left(t_{0}\right)$ is an end point of the curve $C_{i}(t)$. The condition $\mathcal{F}_{1}^{i}>0$ means that the end point $C\left(t_{0}\right)$ is in the interior of the half-plane that contains the convex polygon $P_{k}$ and is bounded by the tangent line of $C_{i}(t)$. Figure 11 shows how the convex hull of multiple rational curves may be computed using the same technique.

\section{CONCLUSION}

In this paper, we have presented an algorithm that computes the convex hull of multiple rational curves in the plane. The input curves may have self-intersections; moreover, they may be open curves. 
In a preprocessing step, we compute a convex polygon that closely approximates the convex hull. By trimming the input curves with respect to this convex polygon, we can make the algorithm output sensitive. The convex hull itself is then constructed using tools for computing point-curve tangents, bi-tangents, and curve-curve tangents.

All examples of this paper were computed within several seconds to several minutes on a SGI machine with a $150 \mathrm{MHz}$ R4000 CPU. The proposed approach is stable and reasonably efficient; it essentially reduces the convex hull problem into that of finding the zero-sets of scalar functions represented in B-spline form. The NURBS representation has nice properties (such as subdivision and convex hull containment) that accelerate the numerical procedure by isolating zero-sets efficiently [5].

\section{ACKNOWLEDGMENTS}

The authors thank anonymous reviewers for their comments which were very useful in improving the expository style of this paper. This research was supported in part by the Fund for the Promotion of Research at the Technion, Haifa, Israel, in part by the Research Fund of Seoul National University, in part by the Korean Ministry of Information and Communication (MIC) under the University Basic Research Program of the year 1999, and in part by the Korean Ministry of Science and Technology (MOST) under the National Research Laboratory Project.

\section{REFERENCES}

1. C. Bajaj and M.-S. Kim, Convex hulls of objects bounded by algebraic curves, Algorithmica 6, 1991, 533-553.

2. M. do Carmo, Differential Geometry of Curves and Surfaces, Prentice-Hall, New York, 1976.

3. D. Dobkin and D. Souvaine, Computational geometry in a curved world, Algorithmica 5, 1990, 421-457.

4. G. Elber, Free Form Surface Analysis Using a Hybrid of Symbolic and Numeric Computation, Ph.D. thesis, University of Utah, Computer Science Department, 1992.

5. G. Elber and M.-S. Kim, Geometric constraint solver using multivariate rational spline functions, in Proc. of ACM Symposium on Solid Modeling and Applications, Ann Arbor, MI, June 4-8, 2001.

6. R. Graham and F. Yao, Finding the convex hull of a simple polygon, J. Algorithms 4, 1983, 324-331.

7. J. Johnstone, A parametric solution to common tangents, in Proc. of Int'l Conf. on Shape Modeling and Applications, Genova, Italy, May 7-11, 2001.

8. D. T. Lee, On finding the convex hull of a simple polygon, Int. J. Comput. Inform. Sci. 12, 1983, 87-98.

9. I.-K. Lee and M.-S. Kim, Primitive geometric operations on planar algebraic curves with Gaussian approximations, in Visual Computing (T. L. Kunii, Ed.), pp. 533-553, Springer-Verlag, Tokyo, 1992.

10. D. Manocha, Solving systems of polynomial equations, IEEE Comput. Graphics Appl. 14, 1994, 46-55.

11. T. Nishita, T. Sederberg, and M. Kakimoto, Ray tracing trimmed rational surface patches, Comput. Graphics 24 (Proc. ACM SIGGRAPH 90), 1990, 337-345.

12. L. Parida and S. Murdur, Common tangents to planar parametric curves: A geometric solution, ComputerAided Design 27, 1995, 41-47.

13. F. Preparata and M. Shamos, Computational Geometry: An Introduction, Springer-Verlag, New York, 1985.

14. A. Schäffer and C. Wan Wyk, Convex hulls of piecewise-smooth Jordan curves, J. Algorithms 8, 1987, 66-94.

15. E. Sherbrooke and N. Patrikalakis, Computation of the solutions of nonlinear polynomial systems, ComputerAided Geometric Design 10, 1993, 379-405. 